\title{
SED-ML web tools: generate, modify and export standard-compliant simulation studies
}

\author{
Frank T. Bergmann ${ }^{1, *}$, David Nickerson ${ }^{2}$, Dagmar Waltemath ${ }^{3}$ and \\ Martin Scharm ${ }^{3}$
}

${ }^{1}$ COS/BioQUANT, Heidelberg University, Heidelberg, Germany, ${ }^{2}$ Auckland Bioengineering Institute, University of Auckland, Auckland, New Zealand and ${ }^{3}$ Department of Systems Biology and Bioinformatics, University of Rostock, Rostock, Germany

*To whom correspondence should be addressed.

Associate Editor: Jonathan Wren

Received on September 23, 2016; revised on November 28, 2016; editorial decision on December 14, 2016; accepted on December 23,2016

\begin{abstract}
Summary: The Simulation Experiment Description Markup Language (SED-ML) is a standardized format for exchanging simulation studies independently of software tools. We present the SED-ML Web Tools, an online application for creating, editing, simulating and validating SED-ML documents. The Web Tools implement all current SED-ML specifications and, thus, support complex modifications and co-simulation of models in SBML and CelIML formats. Ultimately, the Web Tools lower the bar on working with SED-ML documents and help users create valid simulation descriptions.
\end{abstract}

Availability and Implementation: http://sysbioapps.dyndns.org/SED-ML_Web_Tools/.

Contact: fbergman@caltech.edu.

\section{Introduction}

In 2011, the Simulation Experiment Description Markup Language (SED-ML (Waltemath et al., 2011)) was proposed as an exchange format to improve the reproducibility of simulation experiments. Today, SED-ML is acknowledged as a core COMBINE standard (Hucka et al., 2015) and is widely used to exchange simulation experiments in computational biology. SED-ML descriptions typically consist of five major blocks of information: nomination of the model; initialization of the variables; specification of the simulation algorithm; postprocessing of the results; and definition of plots and numerical reports (Bergmann et al., 2015). Libraries to read and write SED-ML are provided by the community and some software tools already consume and export SED-ML files, e. g. BioUML (Kolpakov, 2002), COPASI (Hoops et al., 2006), JWS Online (Olivier and Snoep, 2004) or Tellurium (Sauro et al., 2016). However, tool support is still limited and it remains challenging to derive simulation descriptions given a model, to update a SED-ML file, and to quickly investigate the behaviour of a model that is shared on the internet.

\section{Results}

We developed the SED-ML Web Tools to support users in generating, modifying, simulating, and exporting standard-compliant simulation experiments. The Web Tools are implemented in ASP.NET MVC (http://www.asp.net/mvc) and provide a web based interface to simulation studies using the SED-ML format. They are based on a.NET library called libSedML (http://libsedml.sf.net), that is also available independently.

The SED-ML Web Tools provide an easy-to-use wizard to generate SED-ML files for a model encoded in the SBML format (Hucka et al., 2003). The SBML model can be specified by (i) uploading it to the portal, (ii) providing a link to a web server delivering the model file or (iii) providing a unique resource identifier (URN). Once the model is obtained, the SED-ML Web Tools scan the model's structure for parameters, derive initial values and generate a working SED-ML file using a default configuration (e.g. for a time course simulation). This wizard is only available for SBML encoded models. 


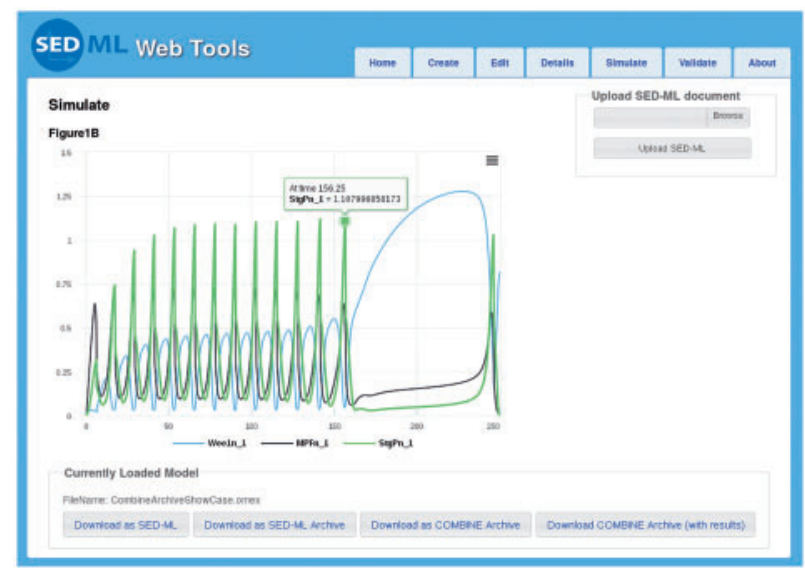

Fig. 1. SED-ML Web Tools importing and then simulating a fully featured COMBINE Archive (Scharm and Touré, 2016). To reproduce, click http://sys bioapps.dyndns.org/SED-ML_Web_Tools/Home/SimulateUrl?url=http:// ndownloader.figshare.com/files/5370005.

An integrated editor allows for revising and modifying the simulation description, e. g. to study an alternative behaviour of the system or to generate plots for different parametrizations. It is available for models encoded in either the CellML or SBML format. The editor supports the SED-ML XML format and a Python-based Script Language (Bergmann, 2011).

Using libSedML, SED-ML files can immediately be simulated on the server and the results are presented in the web browser (Fig. 1). The SED-ML Web Tools run SBML models using RoadRunner (Bergmann and Sauro, 2006), Gillespie and LPsolve. CellML models (Cuellar et al., 2003) are run using CSim (http://get.readthedocs.io). Simulation studies can be exported as (i) standalone SED-ML descriptions; (ii) SED-ML archives; COMBINE archives (Bergmann et al., 2014) (iii) with or (iv) without simulation results. COMBINE archives are zip-based containers to bundle all files relevant for a simulation experiment. The Web Tools are also able to read and understand simulation studies encoded in COMBINE archives. COMBINE archives are extracted, and the encoded simulations are run immediately.

The SED-ML Web Tools can be integrated with other tools. For example, the CombineArchive Web application (WebCAT, Scharm et al. (2014)), an online application to work on COMBINE archives, uses the API of the SED-ML Web Tools. WebCAT provides links to the SED-ML Web Tools to realize instantaneous simulation of studies. A single click on a link launches the SED-ML Web Tools, triggers the download of the corresponding archive from WebCAT's web server, and executes the simulation study encoded in the COMBINE archive. Using the API, simulation studies may even be executed without any human interaction. For example, the M2CAT tool (Scharm and Waltemath, 2015) enriches studies via the addition of simulation results generated using the SED-ML Web Tools. We provide a detailed documentation of the Web Tools' API (sys bioapps.dyndns.org/SED-ML_Web_Tools/Home/API) to demonstrate how to use the Web Tools within your application.

\section{Conclusion}

The reuse of simulation studies is essential in collaborative and responsible research. The SED-ML format and the COMBINE archive are two pioneering approaches to exchange simulation experiments in computational biology and beyond. To support researchers in developing simulation studies that comply with the SED-ML standard, we introduce the SED-ML Web Tools. The API is accessible for external services, for example, to generate simulation descriptions and COMBINE archives remotely, or to execute simulation studies online.

\section{Acknowledgement}

FTB thanks H.M. Sauro for the generous support.

\section{Funding}

This work was funded by the German Federal Ministry of Education and Research (BMBF) as part of the e:Bio SEMS project (FKZ 031 6194) and by the National Institute of General Medical Sciences (grant number GM081070).

Conflict of Interest: none declared.

\section{References}

Bergmann,F. (2011) SED-ML Script Language. Nature Precedings, DOI:10.1038/npre.2011.6105.1.

Bergmann,F. et al. (2014) COMBINE archive and OMEX format: one file to share all information to reproduce a modeling project. $B M C$ Bioinformatics, 15, 369.

Bergmann,F. et al. (2015) Simulation Experiment Description Markup Language (SED-ML) Level 1 Version 2. J. Integr. Bioinf., 12, 262.

Bergmann,F. and Sauro,H. (2006). SBW - a modular framework for systems biology. In: Proceedings of the 38th conference on Winter simulation, pp. 1637-1645. Winter Simulation Conference.

Cuellar,A. et al. (2003) An overview of CellML 1.1, a biological model description language. Simulation, 79, 740-747.

Hoops,S. et al. (2006) COPASI - a complex pathway simulator. Bioinformatics, 22, 3067-3074.

Hucka,M. et al. (2003) The Systems Biology Markup Language (SBML): a medium for representation and exchange of biochemical network models. Bioinformatics, 19, 524-531.

Hucka,M. et al. (2015) Promoting coordinated development of communitybased information standards for modeling in biology: the COMBINE initiative. Front. Bioeng. Biotechnol., 3, 19.

Kolpakov,F. (2002). BioUML-Framework for visual modeling and simulation biological systems. In: Proceedings of the International Conference on Bioinformatics of Genome Regulation and Structure, pp. 130-133.

Olivier,B. and Snoep,J. (2004) Web-based kinetic modelling using JWS Online. Bioinformatics, 20, 2143-2144.

Sauro,H. et al. (2016) Tellurium: a python based modeling and reproducibility platform for systems biology. bioRxiv, 054601.

Scharm,M. et al. (2014). The CombineArchiveWeb application - a web based tool to handle files associated with modelling results. In: Proceedings of the 2014 Workshop on Semantic Web Applications and Tools for life sciences (SWAT4LS).

Scharm,M. and Touré,V. (2016) COMBINE Archive Show Case. Figshare, https://dx.doi.org/10.6084/m9.figshare.3427271.v1.

Scharm,M. and Waltemath,D. (2015) Extracting reproducible simulation studies from model repositories using the CombineArchive Toolkit. In: Lecture Notes in Informatics, volume 3, page e792v1. Workshop on Data management in Life Sciences (Dm4ls), BTW 2015.

Waltemath,D. et al. (2011) Reproducible computational biology experiments with SED-ML - the Simulation Experiment Description Markup Language. BMC Syst. Biol., 5, 198. 